\title{
La fabrique des députés ou l'art de sélectionner les candidats travaillistes et conservateurs aux élections législatives de 2015
}

Crafting the Commons: The Art of Selecting Conservative and Labour

Parliamentary Candidates for the 2015 General Election

Agnès Alexandre-Collier et Emmanuelle Avril

\section{OpenEdition \\ Journals}

Édition électronique

URL : http://journals.openedition.org/rfcb/540

DOI : $10.4000 /$ rfcb. 540

ISSN : 2429-4373

Éditeur

CRECIB - Centre de recherche et d'études en civilisation britannique

\section{Référence électronique}

Agnès Alexandre-Collier et Emmanuelle Avril, «La fabrique des députés ou l'art de sélectionner les candidats travaillistes et conservateurs aux élections législatives de 2015 », Revue Française de Civilisation Britannique [En ligne], XX-3 | 2015, mis en ligne le 01 décembre 2015, consulté le 03 mai 2019. URL : http://journals.openedition.org/rfcb/540 ; DOI : 10.4000/rfcb.540

Ce document a été généré automatiquement le 3 mai 2019.

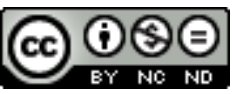

Revue française de civilisation britannique est mis à disposition selon les termes de la licence Creative Commons Attribution - Pas d'Utilisation Commerciale - Pas de Modification 4.0 International. 


\title{
La fabrique des députés ou l'art de sélectionner les candidats travaillistes et conservateurs aux élections législatives de 2015
}

\author{
Crafting the Commons: The Art of Selecting Conservative and Labour \\ Parliamentary Candidates for the 2015 General Election
}

Agnès Alexandre-Collier et Emmanuelle Avril

\section{Introduction}

1 La sélection des candidats est l'une des premières actions à accomplir en vue d'élections : c'est le premier maillon de la chaîne de la délégation, colonne vertébrale du système de démocratie représentative. La sélection des candidats est donc aussi l'une des actions les plus importantes, mobilisant des acteurs ayant des objectifs divers et parfois contradictoires, ce qui nécessite de trouver un compromis entre, d'une part, le contrôle exercé par le centre, et, d'autre part, l'autonomie associée à la démocratie locale ${ }^{1}$. En Grande-Bretagne, cette tension entre le fonctionnement oligarchique des grands partis britanniques et le désir accru de démocratie locale au niveau des sections locales est aussi l'un des principaux problèmes que pose la sélection des candidats.

Cette sélection est l'une (voire la) fonction fondamentale des partis politiques dans les démocraties représentatives ${ }^{2}$ et ce d'autant plus dans un système parlementaire comme la Grande-Bretagne où l'exécutif émane du Parlement. De plus, le mode de scrutin et le fait que près des deux tiers des sièges sont considérés comme «sûrs » (safe) c'est-à-dire acquis à l'un des partis en compétition, expliquent l'équation selon laquelle sélectionner un candidat équivaut à choisir un député. La sélection des candidats détermine non seulement le choix proposé aux électeurs mais aussi la composition de la Chambre des Communes et donc du Gouvernement. Du point de vue des adhérents, l'influence exercée 
sur ce processus de sélection est souvent considérée comme une justification majeure de l'adhésion. Ce processus est donc à la fois une arène permettant l'expression de conflits de pouvoir internes et le moyen par lequel les partis contrôlent le recrutement et le comportement des élus ${ }^{3}$. C'est aussi l'une des procédures les moins visibles et à bien des égards très mystérieuse, rarement étudiée en dehors des États-Unis, et à propos de laquelle on trouve encore peu d'études empiriques car l'accès aux données reste très difficile. Malgré une volonté récente de transparence, il s'agit d'un processus qui reste en effet marqué par le sceau du secret. L'objet de cet article sera précisément de faire la lumière sur les mécanismes qui, au sein des partis conservateur et travailliste, président à la fabrication d'une élite parlementaire, laquelle, dans ce pays plus qu'ailleurs, concentre l'essentiel du pouvoir.

Il s'agit de replacer les décisions stratégiques qui ont présidé à la sélection des candidats pour les élections législatives de 2015 dans une perspective plus générale afin de voir si l'on assiste à une simple consolidation, à des réorientations, voire à des renversements de tendances. Le point commun entre les deux partis réside dans l'origine des réformes entreprises, qui peuvent être qualifiées de top-down en ce sens qu'elles ne proviennent pas de la base, généralement satisfaite de ses prérogatives et de la part d'autonomie qui lui est laissée pour la sélection du candidat local, mais sont à l'instigation exclusive de la hiérarchie du parti. Les réformes sont pour l'essentiel motivées par la nécessité de pallier le déclin inéluctable de l'identification partisane - ou désaffiliation (party dealignement) ${ }^{4}$ qui s'illustre par une baisse du nombre d'adhérents ${ }^{5}$ et une augmentation critique du taux d'abstention aux élections, conjuguée à une volonté croissante de démocratisation interne, dans le cadre de ce que l'on peut qualifier $\mathrm{d}^{\text {' } ~ « i n j o n c t i o n ~ d e ́ l i b e ́ r a t i v e ~} »^{6}$ et qui pèse de plus en plus sur les organisations politiques au sens large. De fait, comme Hazan et Rahat le montrent, la réforme de la sélection des candidats vise à servir simultanément plusieurs objectifs ${ }^{7}$, qui vont de l'accroissement de la diversité des candidats, à l'encouragement de la participation citoyenne, en passant par la sélection de candidats dotés des compétences requises et la limitation des conflits entre la base et le leadership.

En Grande-Bretagne, comme dans la plupart des pays européens, la sélection des candidats est considérée comme la prérogative des sections locales mais cette prérogative reste étroitement contrôlée par le centre, les militants étant régulièrement accusés de sélectionner des candidats peu représentatifs de l'électorat. C'est donc pour répondre à la critique d'une élite parlementaire principalement blanche et masculine, et souvent issue de catégories sociales plus aisées, que les leaders des deux principaux partis (auxquels on pourrait ajouter celui du parti libéral-démocrate même si ce n'est pas l'objet de cet article) ont pris des mesures encourageant une meilleure représentativité en termes de sexe et d'ethnicité. L'introduction des All-Women Shortlists (AWS) chez les travaillistes au début des années 1990 et d'une liste prioritaire - la A-List - chez les conservateurs dès 2006, en est l'exemple le plus probant. Conduites au nom de la "démocratisation », ces réformes, généralement entreprises dans le cadre d'une profonde refonte organisationnelle du parti, ont introduit des procédures délibératives visant à mieux associer les militants, voire l'électorat, aux processus internes de décision. Ce faisant, elles impliquent une remise en cause du principe de l'autonomie des sections locales chez les conservateurs et de celui de la centralité du militant chez les travaillistes. C'est ce qui explique que, dans ces deux partis, ce processus soit devenu l'une des principales sources de tension entre la direction nationale et les adhérents, comme les deux sections de cet article s'efforceront de le montrer. La comparaison de ces deux partis de statut 
comparable au sein d'un même système, tout en faisant ressortir certaines tendances lourdes qui seront soulignées en conclusion, permet d'analyser plus finement la nature de chaque parti et la spécificité de ses luttes internes (en particulier la relation entre leadership et base) ainsi que la manière dont chacun gère ses relations avec les électeurs, ce que les études comparatives plus vastes, dominantes à l'heure actuelle sur ce sujet, ne permettent pas d'envisager.

\section{La sélection des candidats conservateurs}

5 La nature même du parti conservateur explique en grande partie les modes de sélection des candidats parlementaires, leurs failles et les réformes introduites en $1998^{8}$ pour améliorer le dispositif. Le parti a longtemps été présenté comme l'idéal type du modèle oligarchique centré autour du leader et d'une poignée de dirigeants concentrant l'essentiel du pouvoir. L'image du «cercle magique » appliquée à l'équipe dirigeante a, jusqu'en 1965, illustré une structure peu démocratique, entièrement tendue vers l'émergence d'une équipe pressentie comme capable de prendre les rênes du pouvoir. D'une certaine manière, la sélection des candidats parlementaires reproduisait, au niveau local, cette oligarchie, en fabriquant une élite parlementaire démographiquement et socialement semblable à l'équipe dirigeante mais peu représentative de sa base électorale et militante. Malgré ce mimétisme, la structure même du parti permettait aux sections locales de jouir d'une certaine autonomie et, jusqu'en 1998, on peut dire que les sections locales et le siège entretenaient une relation assez distendue, chaque composante conservant sa propre sphère d'influence. Cette relation a fait l'objet d'une abondante littérature, Peter Mair ${ }^{9}$ évoquant par exemple, en 1994, un fonctionnement qu'il appelle " stratarchique " du parti conservateur, c'est-à-dire permettant aux différentes composantes de fonctionner comme des strates disposant, chacune, de compétences et de prérogatives propres. Plus explicitement, Kenneth Carty ${ }^{10}$ évoque le modèle du " partifranchise " permettant de clarifier la relation entre le siège et les sections locales en comparant ces dernières à des franchises du parti, dont le rôle est bien de diffuser et vendre la "marque » conservatrice mais qui disposent d'une grande autonomie, voire fonctionneraient de manière semi-autarcique comme en témoignent notamment leurs modes de sélection des candidats.

Jusqu'en 1998, la sélection des candidats est un processus relativement uniforme dans lequel le siège et les sections locales interviennent de manière alternée et équivalente, à toutes les étapes du processus. Pour pouvoir participer au processus, les postulants doivent préalablement figurer sur une « liste approuvée " par la direction nationale, après avoir subi une série de tests. Cette étape essentielle est celle du «Parliamentary Selection Board » (PSB) qui s'inspire de la procédure d'entraînement des officiers de l'armée telle qu'elle se pratique dans l'école militaire de Sandhurst. La liste élaborée à la suite du PSB est ainsi décrite comme "un menu fixe ${ }^{11}$ dans lequel les sections locales sont invitées à piocher. Chaque section locale peut alors recevoir une trentaine de postulants approuvés, à partir de laquelle sera établie une liste courte de trois ou quatre postulants présélectionnés à l'issue d'un jeu d'interviews conduits en alternance par le siège et la section locale. La dernière étape ("general meeting ») rassemble ensuite tous les membres actifs de la section locale et les adhérents de la circonscription, présents lors de l'assemblée, pour élire celui qui deviendra leur candidat. 
7 Ce dispositif, qui permet aux circonscriptions de jouir d'une autonomie relative par rapport au siège, a néanmoins produit ses propres effets pervers, à savoir une sélection nettement biaisée en faveur d'un même type de candidat blanc, masculin et issu d'un système éducatif sélectif et privé. Autrement dit, jusqu'en 1998, le manque de représentativité des candidats conservateurs et de diversité au sein du groupe parlementaire est perçu comme le résultat d'une sélection trop ancrée dans les sections locales et presque entièrement laissées aux mains des militants sans contrôle véritable par le siège.

C'est dans cet esprit que vont être conduites les réformes organisationnelles introduites par le leader de l'époque William Hague à la suite de l'humiliant revers électoral de 1997. L'objectif de ces réformes était double: officiellement, il s'agissait de démocratiser l'organisation partisane en introduisant des dispositifs plus délibératifs entre les dirigeants et la base. Ainsi les adhérents furent-ils invités à participer à l'élection du leader et à prendre part plus activement au processus de décision par des consultations plus régulière sous forme de "focus groups » et de référendums internes. Officieusement, il s'agissait pour le siège de mieux centraliser les décisions. Cette centralisation fut rendue possible par l'introduction de nouveaux statuts permettant d'unifier l'organisation désormais chapeautée par un bureau politique («Party Board») ayant un droit de regard sur toutes les composantes du parti, en particulier les sections locales. En matière de sélection des candidats, les réformes de 1998 n'envisageaient cependant que des ajustements à la marge, comme le remplacement du " Parliamentary Selection Board " par un «Parliamentary Assessment Board " prévoyant un mode de recrutement fondé sur des compétences de communication, de gouvernance et de motivation, et calquée non plus sur le modèle de l'armée mais sur celui de l'entreprise. Néanmoins l'un des effets indirects des réformes de 1998 a été d'accroitre la centralisation et le contrôle sur la sélection des candidats puisque le siège est désormais présent, de manière presque systématique, à chaque étape de la procédure.

\section{Les mécanismes de sélection}

9 Ainsi, depuis 1998, la procédure de sélection des candidats, justifiée par une volonté d'améliorer la représentativité, révèle une centralisation accrue de la structure organisationnelle.

\section{Tableau 1}

\section{Standard Conservative Candidate Selection Process (2010)}

a) Nominated by local associations Apply via CV

CCHQ sift

b) Parliamentary Assessment Board (PAB)

c) Acceptance onto Approved List / A-List

d) Constituency CV Sift

e) Long List

f) Selection Committee Interviews

g) Shortlisting by Selection Committees

h) General Meeting to select committee 


\section{Source : Williams et Paun (2011), p. 1}

Le processus est agencé autour de plusieurs étapes qui, jusqu'en 2003, sont les mêmes pour toutes les sections locales. Il reste très semblable à ce qu'il était avant 1998. Pour pouvoir se présenter dans la circonscription de leur choix, les postulants doivent, au préalable, être approuvés par le siège. Pour figurer sur la liste approuvée par le siège (« approved list »), ils doivent d'abord envoyer leur CV au siège puis subir une journée de tests lors du Parliamentary Assessment Board. A l'issue de cette étape, il reste une trentaine de candidats "approuvés » qui sont ensuite interviewés alternativement par la section locale et le siège à partir de la liste préalable qui passe ainsi d'une trentaine de personnes à trois ou quatre postulants. Ce sont sur ces postulants que vont se prononcer les adhérents de la section réunis lors d'une assemblée (" general meeting ») organisée dans la section locale.

3 Les réformes de 1998 ont toutefois fait apparaître un rôle accru du siège (CCHQ) par rapport à la procédure antérieure. Le siège intervient désormais à chaque étape de la procédure, y compris dans celles qui étaient jusqu'à présent réservées aux sections locales, qu'il s'agisse du tri des CV opéré par le Candidates' Team au CCHQ, du Parliamentary Assessment Board qu'il organise, des entretiens qu'il mène en parallèle avec les sections locales, de l'approbation des trois ou quatre candidats choisis par la circonscription, voire dans l'ultime étape où un représentant du siège est désormais présent pour scruter, arbitrer et contrôler le déroulement de cette réunion finale. Non seulement la sélection des candidats s'avère-t-elle encore plus centralisée, mais le problème de la sousreprésentation des femmes et des minorités ethniques n'est pas réglé pour autant. Les réformes de 1998 sont vite accusées de révéler la véritable nature - oligarchique - de l'organisation sous couvert de démocratisation, en propulsant le leader à la tête d'une immense structure désormais unifiée et en lui permettant de garder la mainmise sur la moindre composante, $\mathrm{y}$ compris les sections locales jadis très autonomes.

Dès 2005, deux dispositifs vont donc être introduits pour corriger les failles des réformes de 1998, visant à améliorer la représentativité du groupe parlementaire et compenser, cinq ans après la publication de A Fresh Future for the Conservative Party, la centralisation accrue de l'organisation partisane. Une liste parallèle - ou A-list - est proposée aux sections locales qui ont désormais le choix entre les postulants de la liste approuvée et ceux de la A-list. Cette liste prioritaire comprend une centaine de candidats dont $50 \%$ doivent être des femmes et une proportion significative de minorités ethniques. Or les critères permettant aux postulants de figurer sur cette liste sont très opaques et les postulantes - essentiellement des femmes -, dont les compétences sont alors remises en cause, sont rapidement accusées d'avoir été parachutées par le siège sans avoir à subir de tests de sélection. La même année sont introduites dans les sections locales qui en font la demande les premières primaires ouvertes permettant aux électeurs qui s'inscrivent de participer au vote final pour sélectionner le candidat. Ainsi la procédure reste la même à l'exception de la dernière réunion jadis réservée aux adhérents et désormais ouverte aux électeurs. Dès l'élection de David Cameron à la tête du parti, ces dispositifs innovants vont être rapidement étendus et vendus par le dirigeant comme un instrument de la modernisation qu'il compte opérer au sein du parti. De 2006 à 2009, ce sont ainsi 116 primaires qui sont organisées dans les sections locales. Il s'agit uniquement d'assemblée primaires ("primary meetings ») réunissant adhérents et électeurs inscrits. En mai 2009, deux primaires postales sont organisées à Totnes et Gosport à la suite du scandales des notes de frais et destinées à sélectionner le candidat conservateur avant les élections 
partielles. Avant les élections de mai 2015, seules 26 primaires ont été organisées et le processus, encensé par David Cameron en 2010, s'est considérablement essoufflé, n'ayant pas permis au parti d'être plus représentatif ni de fonctionner plus démocratiquement. Le dispositif a été en effet, une fois de plus, rigoureusement contrôlé par le centre, comme si les mesures de démocratisation - qui évoquent l'idée d'une ouverture horizontale vers la société civile - devaient nécessairement s'accompagner d'un mouvement vertical de centralisation qui chercherait à en limiter les effets pervers. Ainsi les primaires sont-elles restées un dispositif expérimental et voué à l'échec en raison d'un taux de participation toujours très faible ${ }^{12}$, d'un résultat équivalent à celui de la sélection traditionnelle en termes de représentativité et de ses effets présumés sur le comportement parlementaire du député issu d'une primaire ${ }^{13}$. Symboliquement, les primaires ont toutefois abouti à des résultats intéressants dans certaines circonscriptions, comme les élections de 2015 ont pu le laisser entrevoir. Ainsi, selon nos estimations, sur les 26 primaires organisées avant 2015, 19 ont abouti à l'élection d'un candidat conservateur. Si 12 de ces circonscriptions étaient traditionnellement acquises au parti conservateur (safe seats), la quasi-totalité des primaires organisées dans des circonscriptions marginales (4) ou acquises aux LibérauxDémocrates (2) ont permis de faire basculer la circonscription dans le camp conservateur bien qu'il soit difficile de savoir si ce résultat est un effet de la primaire ou le produit d'autres facteurs. Quoi qu'il en soit, trois de ces circonscriptions, qui ont été très médiatisées, méritent d'être signalées: à Twickenham où le ténor du parti libéraldémocrate, Vince Cable, a perdu son siège (très sûr) au profit de la candidate conservatrice Tania Mathias et à Morley and Outwood où Ed Balls, le challenger présumé de Ed Miliband à la tête du parti, a perdu son poste au profit d'Andrea Jenkyns, les deux candidates conservatrices avaient été sélectionnées par une primaire ouverte. A Rochester and Strood, la candidate conservatrice, Kelly Tolhurst, qui a évincé l'un des deux députés UKIP, Mark Reckless, qui avait quitté le parti conservateur en 2014 pour rejoindre le parti de Nigel Farage, venait d'être sélectionnée par une vaste primaire postale organisée par le parti à la suite de la défection de Reckless. Même si l'échantillon de 26 primaires ouvertes ne permet pas d'en tirer des conséquences significatives, il n'en reste pas moins que ces quelques expériences locales révèlent l'importance symbolique de la primaire sur trois plans : premièrement par la façon dont elle a permis de porter sur le devant de la scène certains candidats (comme Andrea Jenkyns, précédemment citée, très présente sur les réseaux sociaux) avant même les élections de 2015, deuxièmement en court-circuitant en quelque sorte la progression de partis comme le UKIP par l'introduction d'un dispositif qui à bien des égards peut être considéré comme l'un des instruments du populisme qu'ils affichent, troisièmement en contournant les adhérents pour permettre aux députés d'établir une connexion directe avec les électeurs, renforçant par la même la centralisation autour du leader.

\section{Les effets : profil des candidats et des élus conservateurs ${ }^{14}$}

S'il est difficile de mesurer l'effet de la A-list et des primaires ouvertes sur la représentativité du groupe parlementaire élu en mai 2015, il n'en reste pas moins que ces élections ont été saluées comme des élections historiques en matière de diversité sexuelle et ethnique. L'évolution est particulièrement frappante chez les conservateurs dont les députés comptent traditionnellement moins de femmes et de minorités ethniques. Il a pu être également étonnant de trouver, parmi les candidats conservateurs issus de l'immigration, des profils atypiques, à l'image de Amandeep Singh Boghal, candidat sikh 
portant turban et longue barbe et se présentant dans une circonscription d'Irlande du Nord ou des profils nouveaux, comme Alan Mak, premier candidat conservateur d'origine chinoise et sélectionné à l'issue d'une vaste primaire ouverte - «à l'américaine » précise le site de la section - dans la circonscription de Havant. On dénombre ainsi 67 femmes élues, soit $20 \%$ du groupe parlementaire (contre $16 \%$ en 2010) sur 164 candidates sélectionnées par le parti, soit $25 \%$ du total. Par ailleurs, 17 députés issus de minorités ethniques ont été élus soit 5\% du groupe (comparé à 3,5\% en 2010), sur un total de 62 minorités (BAME ou " Black and Asian Minority Ethnic »people) qui avaient été sélectionnées, soit 9,5\% de l'ensemble des candidats conservateurs. Malgré ce renouvellement, on dénombre peu de nouveaux députés élus pour la première fois (19\%) contre $81 \%$ de réélus, à la différence des travaillistes. Si les nouveaux députés conservateurs sont aussi sensiblement plus âgés (50,2 ans en moyenne contre 47,8 en 2010), leur profil social est cependant remarquablement constant puisque $71 \%$ des députés conservateurs sont issus du milieu des affaires, de la finance et du droit, lesquels représentent respectivement $29 \%, 25 \%$ et $17 \%$ des députés du parti. On trouve toujours une prédominance de chefs d'entreprise mais aussi une diminution nette d'universitaires ( $2 \%$ contre $8 \%$ en 2010 ). On note aussi une proportion légèrement en baisse de députés issus des relations publiques, à l'image de leur dirigeant David Cameron (12\% en 2015 contre 15\% en 2010). Le changement le plus significatif provient de leur formation: on trouve en effet plus de députés issus du système des universités les plus prestigieuses comme Oxford ou Cambridge que lors des élections de 2010 (soit $45 \%$ contre 32\%) mais un peu moins d'entre eux proviennent des écoles privées ( $52 \%$ contre $54 \%$ en 2010 ). Autrement dit, si le visage du nouveau groupe parlementaire est aujourd'hui plus féminin et coloré que le précédent, il reste remarquablement constant en termes d'origines sociales et de catégories socioprofessionnelles.

La sélection des candidats conservateurs révèle un effort constant d'ouverture vers la société civile. Le parti conservateur fait ainsi figure de pionnier dans l'utilisation de primaires ouvertes qui s'appuient sur des électeurs dont on peut supposer qu'ils manifestent un intérêt pour le parti ou au moins pour le candidat si celui-ci est déjà connu dans la circonscription ou dispose d'un fort ancrage local. Mais derrière ce dispositif transparaît une stratégie de la part du parti pour rehausser son image de marque. Au-delà de la sélection des candidats, on pourrait même y voir l'un des instruments de communication de David Cameron prêt à toutes les innovations pour diffuser sa vision d'une "ère post-bureaucratique $»^{15}$ selon laquelle le pouvoir doit être distribué au peuple. Dans une certaine mesure, ce dispositif incarne aussi l'inauguration d'une nouvelle pratique démocratique plus délibérative à l'échelle du parti et du pays tout entier, qu'illustre aussi l'extension de la pratique référendaire comme nouveau mode de décision gouvernementale, à l'instar du référendum sur l'indépendance écossaise de septembre 2014 ou de celui annoncé sur le maintien du pays dans l'Union européenne. Derrière ce nouveau mode de sélection des candidats se cache aussi une volonté paradoxale de mieux contrôler le type de candidat traditionnellement choisi, l'arrivée massive des femmes et des minorités ethniques ne correspondant en réalité pas au choix des militants de base. Derrière ce dispositif se cache enfin une volonté nette du parti d'étendre sa base de soutiens. La mise en place d'un réseau de "registered supporters » Team 2015 - par le président du parti Grant Shapps, va de pair avec l'ouverture du processus de sélection à des électeurs dont on peut supposer qu'une fois inscrits dans les registres de la section locale, ils feront partie de ce vaste réseau sur lequel le parti 
envisage de compter désormais, pour pallier la baisse radicale du nombre d'adhérents. Moyennant une participation de $1 £$, ces supporters ${ }^{16}$ ont eu la possibilité d'apporter leur aide sur le terrain, à la campagne électorale, suppléant ainsi les militants dans un domaine qui leur était réservé. Mais en dépossédant aussi les adhérents du parti de leur principale prérogative - le monopole de la sélection du candidat local - le parti risque également de perdre, à terme, sa principale source de financement.

\section{La sélection des candidats travaillistes}

$\mathrm{Au}$ parti travailliste comme au parti conservateur, la sélection des candidats a longtemps été la prérogative des sections locales. Le contrôle du processus par les sections locales avait même été renforcé en 1981 avec l'adoption de la "re-sélection obligatoire " (" mandatory reselection») des députés sortants ${ }^{17}$, qui avait constitué une victoire de l'aile gauche du parti, alors en pleine ascension. Depuis le début des années 1990, cependant, les réformes introduites visant à la fois à "démocratiser » le processus (c'est-à-dire à reconnecter le parti avec son électorat dans une quête de légitimité) et à encourager la diversité (afin d'obtenir une meilleure représentation de l'électorat) ont été impulsées de haut en bas et se sont accompagnées d'un contrôle accru de la direction nationale sur le processus. En 1993, l'adoption du vote par tête (OMOV - «one member one vote») pour la sélection des candidats, qui mettait un terme à un vote bloqué syndical pesant pour $40 \%$ des voix au sein des collèges électoraux locaux ${ }^{18}$, reflétait un effort plus général pour réduire le poids des syndicats dans les processus décisionnels du parti et réduire l'influence des militants (considérés comme trop radicaux) sur le processus. A partir de 1995, après l'élection de Blair à la tête du parti et dans la perspective des élections législatives à venir, on observe un changement d'approche plus marqué. Avec le New Labour, la priorité est désormais donnée à la sélection de candidats compétents et capables de projeter une image centriste et moderne et qui, une fois élus, soutiendraient Blair. L'objectif des réformes était donc de rééquilibrer un groupe parlementaire dont le centre de gravité était, aux yeux du leadership, situé trop loin sur la gauche ${ }^{19}$. Tout est alors mis en œuvre pour faire émerger les « bons » candidats, c'est-à-dire ceux dont le profil est conforme aux objectifs stratégiques de la direction nationale, dans les sièges gagnables, ce qui passe par une mainmise du centre sur le processus de sélection, par le biais de réformes destinées à accroître l'ouverture et la diversité, dont la plus marquante a été l'introduction des «All Women Shortlists » (AWS) ${ }^{20}$.

\section{Les mécanismes de sélection}

Contrairement à ce qu'on trouve chez les conservateurs, la procédure de sélection est très strictement encadrée, codifiée et unifiée. Les documents internes tels que le NEC Procedural Guidance2013 et, sous une forme plus condensée, le Rule Book 2014, stipulent dans le menu détail les principes et objectifs, les rôles respectifs du Comité exécutif national ( National Executive Committee » - NEC) et de la section locale, la composition du comité de sélection, les critères d'éligibilité des candidats, le sélectorat et les critères d'éligibilité des adhérents. A noter qu'il est cependant possible de se dispenser de la procédure en cas d'urgence, lorsque le calendrier l'impose, comme c'est le cas lorsqu'un député sortant se retire à une date tardive ou bien simplement lorsque que le NEC considère qu'il est dans 
l'intérêt du parti de le faire ${ }^{21}$. Cette procédure abrégée ouvre grand la porte aux tentatives de parachutages.

Une caractéristique majeure du processus est d'être contrôlé plus ou moins directement de bout en bout par le Comité exécutif national, par le biais notamment du comité de sélection piloté par un représentant $\mathrm{du} \mathrm{NEC}{ }^{22}$. La composition de ce comité est soumise à des règles strictes en vue de refléter l'équilibre des forces au sein de la section locale et de répondre à des critères de représentativitée 3 .

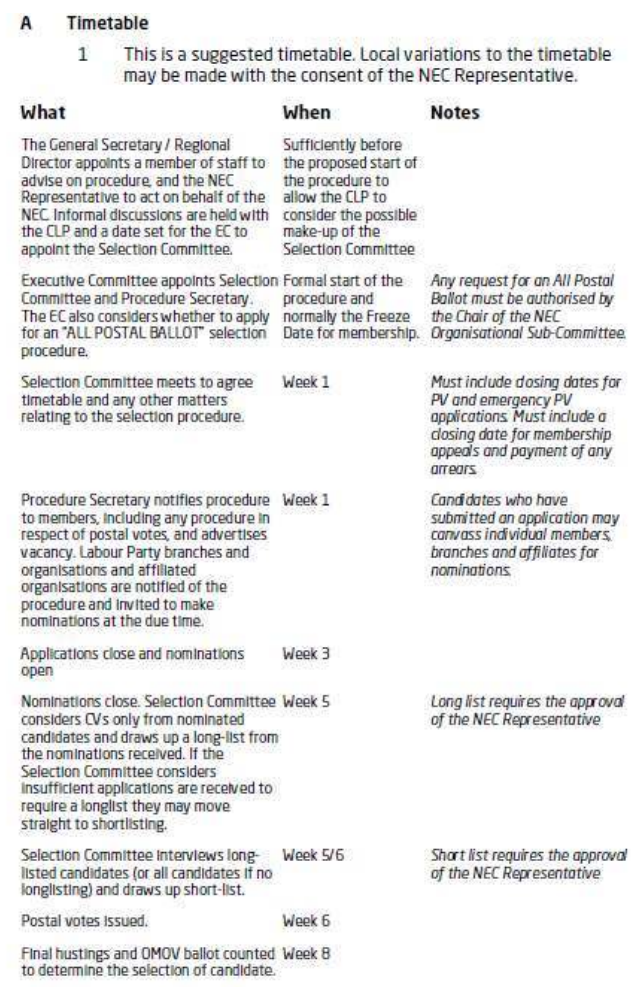

Source: The Labour Party, NEC Procedural Guidance, 2013, p. 13.

Bien que, chez les travaillistes, le principe d'une liste nationale de candidats établie par le NEC au sein de laquelle les sections locales peuvent choisir leur candidat ait une longue histoire ${ }^{24}$, la stratégie déployée au plan national au milieu des années 1990, en vue d'accroître la représentativité du groupe parlementaire, a impliqué un rôle plus actif du NEC. Même si la mainmise de la direction nationale semble, depuis 2010, un peu moins directe, le rôle du NEC se définit en termes d'une "supervision» de l'ensemble du processus de sélection afin d'atteindre les objectifs stratégiques de la direction nationale ${ }^{25}$ . Les principes régissant la constitution de la «short-list $»^{26}$, étape cruciale du processus, visent à réconcilier des objectifs parfois contradictoires : d'une part la promotion de la diversité et d'autre part la nécessité de faire émerger des candidats «compétents $»^{27}$. En outre, le NEC se réserve un droit de veto (exercé à plusieurs reprises) ${ }^{28}$ et a le dernier mot en cas de conflit ${ }^{29}$.

31 En outre, alors que jusque-là l'accent avait été principalement mis sur des caractéristiques telles que le sexe et l'origine ethnique, lors du congrès annuel de 2012, les règles de diversité ont été élargies en vue d'encourager également la sélection de candidats handicapés ou issus de la communauté gay et lesbienne, et, dans le même temps, d'obtenir une meilleure représentation de la classe ouvrière ${ }^{30}$. L'action du NEC vise essentiellement à agir au niveau de l'offre ${ }^{31}$, en encourageant et soutenant les candidats 
potentiels ayant ce type de profil, notamment par le biais du programme de formation intitulé «Future Candidates» qui a permis l'élection, le 7 mai 2015, de 53 députés travaillistes sans expérience (soit $21 \%$ du parti parlementaire). Cela permet de faire émerger un nouveau type de candidats ayant moins d'ancienneté dans le parti, dotés de ressources personnelles plus limitées (une candidature réclame un investissement financier personnel non négligeable) et ne bénéficiant pas du soutien d'un syndicat. Du point de vue de la direction nationale, ces candidats moins expérimentés sont aussi plus dociles et susceptibles de constituer un groupe parlementaire plus homogène et légitimiste $^{32}$. On voit ainsi l'effet paradoxal de mesures destinées à encourager la diversité, qui relèvent donc d'une démocratisation au sens d'une meilleure représentativité des candidats et du groupe parlementaire, mais qui réduisent encore un peu la marge de manœuvre des sections locales, contribuant donc à éroder le degré de démocratie interne.

Une autre dimension cruciale est la composition du « sélectorat $~_{33}$. Le passage au vote par tête a mis un terme au vote bloqué syndical, mais n'empêche pas les syndicats d'avoir une influence sur le processus, ce qui est parfois source de conflit avec la direction nationale ${ }^{34}$ . Il s'agit d'une modification majeure qui affecte la nature même de l'organisation, ce qu'elle représente et la façon dont elle fonctionne ${ }^{35}$. Des règles strictes d'éligibilité fixent les conditions à remplir pour prendre part au scrutin: d'une part le droit de vote est réservé aux seuls adhérents ${ }^{36}$, d'autre part une condition d'ancienneté est imposée ${ }^{37}$, ce afin de réduire les distorsions résultants d'éventuelles vagues d'adhésions massives juste avant le scrutin. Ces conditions peuvent sembler anodines, mais, comme pour toute procédure, le «diable est dans les détails ». C'est pourquoi les désaccords ne sont pas seulement anecdotiques, mais au contraire le révélateur de tensions intraorganisationnelles fondamentales ${ }^{38}$. C'est ce qu'illustre le cas des sections locales de Falkirk et de Halifax, qui ont eu le plus de visibilité dans les médias, où les conflits ont justement porté sur l'éligibilité des votants. Dans le cas du «scandale » de Falkirk, en 2013, le syndicat UNITE avait été accusé d'avoir payé les cotisations d'une partie de ses membres afin de peser davantage sur le résultat final. Même si l'enquête policière a finalement statué que la pratique n'avait rien d'illégal, le retentissement médiatique a été tel qu'il a précipité l'adoption, en mars 2014, des réformes du mode d'affiliation des membres des syndicats dans le parti ainsi que le mode d'élection du leader travailliste. Halifax a été, au printemps 2015, le théâtre d'un autre bras de fer entre ce même syndicat - dont la candidate était celle-là même qui avait été suspendue à Falkirk, puis réintégrée et le NEC, accusé de vouloir lui faire barrage à tout prix. Suite à une plainte de la députée sortante auprès du NEC, un comité de sélection spécial a été constitué sous la houlette de Harriet Harman. Après la suspension de 134 membres ayant récemment adhéré, en vertu d'une nouvelle règle imposant une ancienneté d'au moins un an au lieu de six mois, c'est la candidate légitimiste qui a été élue à une courte majorité. L'aile gauche du parti dénonce le manque de transparence concernant les raisons véritables de la "dé-sélection » de candidats par le NEC au motif d' «irrégularités ». La sélection des candidats cristallise ainsi les tensions entre leadership et syndicats, et fait l'objet de fréquentes accusations croisées de parachutages et de manipulation ${ }^{39}$.

33 Il faut donc souligner le fossé entre les règles formelles et leur mise en pratique. L'introduction d'OMOV, qui visait essentiellement à accroître l'autonomie stratégique de la direction nationale $e^{40}$, rend en réalité la manipulation du processus dans son ensemble plus difficile ${ }^{41}$ (les tentatives échouent parfois de manière spectaculaire, comme on l'a vu 
pour les élections à la Mairie de Londres), tout en ouvrant la porte à des ajustements " discrets " de certains aspects du mécanisme, comme celles portant sur les règles d'éligibilité des votants ${ }^{42}$. Il faut en outre souligner le rôle majeur des permanents du parti au niveau régional ( regional organisers »), courroies de transmission des décisions stratégiques de la direction nationale, qui œuvrent en sous-main à orienter les choix des sections locales, par le biais de pressions informelles - mais néanmoins très pressantes sur des membres clés de ces dernières. Il est impossible de mesurer avec précision l'étendue, la fréquence ou l'effet de ces pressions, mais elles existent indéniablement ${ }^{43}$. Les plaintes adressées au NEC ont d'ailleurs souvent trait aux techniques employées par les dirigeants (liste d'adhérents fournies à l'avance à certains candidats, irrégularités concernant les votes postaux etc.). Cet aspect requiert des recherches empiriques plus poussées car les cas rendus publics sont rares.

\section{Les effets : profil des candidats et des élus travaillistes ${ }^{44}$}

Sur les 631 candidats travaillistes sélectionnés en vue des élections du 7 mai $2015^{45}$, 34\% étaient des femmes. Le fait que $53 \%$ de ces candidates aient été sélectionnées dans des sièges gagnables explique un très bon taux de conversion entre le pourcentage de candidates et le pourcentage d'élues, en dépit des pertes sèches ${ }^{46}$ : avec 99 femmes élues aux Communes (soit $41 \%$ du groupe parlementaire contre $31 \%$ en 2010), le parti travailliste continue de se distinguer. On voit comment les AWS, au demeurant toujours controversées ${ }^{47}$, ont institué une égalité des chances entre les hommes et les femmes dans les sièges gagnables et ainsi permis de constituer une réserve de députées solidement implantées à Westminster, ce que les quotas en place depuis les années 80 n'avaient pas permis de faire. En l'absence de loi sur la parité, la discrimination positive pratiquée par le parti le distingue clairement de ses rivaux. En comparaison, la promotion des candidats issus des minorités ethniques (on pense par exemple au programme « Operation Black Vote »), pour lesquels c'est l'encouragement plutôt que la discrimination positive qui prévaut, est restée plus limitée. Toutefois on observe en 2015, grâce à la sélection de candidats dits «BAME» dans des sièges gagnables ( $9 \%$ des candidats, et $13 \%$ dans des sièges gagnables) un bon taux de conversion avec l'élection de 23 députés avec ce profil, soit $10 \%$ du groupe parlementaire travailliste (contre 5,8\% en 2010), proche du profil de la population (12,1\% de BAME selon les chiffres du recensement de 2011). La section locale d'Edmonton a même établi une "short-list » entièrement composée de femmes «BAME ». La progression est donc très nette.

Concernant l'origine sociale, les disparités perdurent en dépit des déclarations d'intention, puisque la proportion de députés travaillistes issus des écoles privées sélectives est stable, à $12 \%$, de même que ceux ayant suivi des études dans les universités prestigieuses d'Oxford et Cambridge (17\%). En termes de profession, le groupe parlementaire travailliste se distingue par une proportion plus élevée de députés issus du mouvement syndical (15\%, chiffre en baisse par rapport à 2010 où l'on en comptait 18\%) et du secteur associatif ( $15 \%$ également). Cependant il faut souligner que la proportion de députés travaillistes issus du milieu des affaires a doublé (14\%, contre $7 \%$ en 2010$)$ alors que la sous-représentation de la classe ouvrière s'accentue, avec seulement $7 \%$ de travailleurs manuels ( $9 \%$ en 2010) contre $12 \%$ issus des professions juridiques (un point de plus qu'en 2010) et $10 \%$ du secteur des médias. Pour être vraiment représentatif, le PLP devrait compter plus de $50 \%$ de députés issus de la classe ouvrière (un sondage réalisé 
par le British Election Survey en 2013 indiquait que 61\% de la population se considère comme "working class ») $)^{48}$. Un autre trait particulièrement marquant est la proportion croissante de "professionnels» de la politique (29\%), de même que la proportion de députés élus pour la première fois $(21 \%)$ et la surreprésentation au sein de cette dernière catégorie de femmes (65\%) et de minorités ethniques (15\%). Ces éléments confirment les tendances identifiées en 2010 et l'idée d'une convergence entre les principaux groupes parlementaires.

L'évolution des méthodes de sélection des candidats parlementaires travaillistes doit se concevoir dans le cadre plus large des réformes organisationnelles impulsées à partir du début des années 1990, car elle fait écho à d'autres changements tels que la réforme de l'élection du leader, la réduction progressive du poids des syndicats dans les différentes instances du parti, et la mise en place de réseaux de supporters. Toutes ces réformes vont dans le sens d'une individualisation du vote, accompagnée d'une centralisation croissante 49. Comme chez les conservateurs, la tendance lourde depuis le début des années 90 a donc été celle d'un contrôle accru de la direction nationale sur le processus de sélection. L'adoption du vote par tête (OMOV) permet en effet au NEC d'intervenir à toutes les étapes du processus, ce qui génère des tensions préoccupantes entre la base et le leadership, mais permet dans le même temps de préserver la cohésion au niveau du groupe parlementaire dont la composition est stratégiquement orientée de façon à ce que les députés puissent jouer un rôle de soutien au leadership. Au plan local, la sélection des candidats parlementaires travaillistes obéit aujourd'hui à des logiques contradictoires, puisqu'on observe une tension entre les objectifs des syndicats soucieux de favoriser une meilleure représentation de la classe ouvrière, et ceux d'un leadership dont la priorité a surtout été d'encourager la représentation des minorités, pour la plupart issus des classes moyennes. Les perspectives d'évolution concernent le sélectorat, probablement voué à s'élargir dans une quête de représentativité, ce qui pose la question du rôle et des droits devant être octroyés aux "registered supporters ", déjà invités à participer à l'élection du prochain leader, par rapport aux adhérents s'acquittant d'une cotisation et - pour certains - participant activement à la vie de la section locale. Pour l'heure, la démocratisation du processus de sélection reste très relative puisque le choix proposé aux membres des sections locales travaillistes est à la fois filtré et fermement encadré par les instances nationales.

\section{Conclusion}

37 La sélection des candidats aux élections de mai 2015 a modifié le visage de la Chambre de la Communes qui s'avère aujourd'hui plus féminin, plus coloré et sans doute plus moderne qu'il ne l'a jamais été, ou, comme on dit en anglais, «less stale, pale and male ». Ce résultat, est, comme nous l'avons vu, le fruit d'un processus complexe qui a subi d'importantes modifications dans le cadre de réformes organisationnelles plus larges. Parmi les changements les plus notables, on note une ouverture plus nette vers la société civile qui va de pair avec ce que Katz et Mair ${ }^{50}$ ont appelé la cartellisation des partis, ou la transformation de l'objet partisan en une entité qui aspire à se confondre avec la société et dont les frontières, notamment entre les adhérents et les non-adhérents, deviennent de plus en plus poreuses. L'indice le plus net de cette transformation est l'introduction de primaires pour la sélection des candidats. Qu'elles soient fermées, dans le cas des travaillistes (c'est-à-dire limitées aux seuls adhérents), ou ouvertes (aux électeurs) chez 
les conservateurs, l'idée commune est de multiplier les procédures délibératives et d'associer le plus possible la base (militante ou électorale) aux processus de décision. Au chapitre des convergences entre les deux partis, on note aussi une utilisation de catégories nouvelles d'individus qui ne sont pas financièrement affiliés au parti: les supporters. Ces points communs signalent également un effet de mimétisme entre les deux grands partis majoritaires et un mouvement de convergence vers le modèle de particartel annoncé par Katz et Mair. Ainsi est-il permis de penser que ces "registered supporters ", qui peuvent déjà participer à la sélection du candidat conservateur dans certaines circonscriptions et peuvent désormais participer à l'élection du leader travailliste, pourraient, à terme, dans ce parti, se voir également dotés du droit de participer à la sélection des candidats.

Pour autant, le fait que le processus de sélection soit désormais plus ouvert à la société civile ne signifie pas qu'il soit devenu plus transparent. Il subsiste en effet un profond hiatus entre les motivations officielles et l'agenda secret des partis. Derrière l'apparente démocratisation, le processus reste en effet étroitement contrôlé et surveillé par la direction nationale. Tout semble indiquer que l'intention est de garder la mainmise sur les sections locales en contournant les militants sous le prétexte qu'ils ont tendance à sélectionner des candidats peu représentatifs de la société. Par le bais des primaires ouvertes, le parti conservateur en vient même à priver les adhérents de leur principale prérogative tout en continuant à s'appuyer sur eux pour la campagne de terrain et le financement $\mathrm{du}$ parti. Le parti travailliste, on le voit, évolue dans la même direction. Ainsi, sous couvert de diversité, l'objectif implicite dans les deux partis serait de produire un type de député plus malléable. La diversité et la recherche d'un profil différent du type de député traditionnellement majoritaire dans ces partis pourrait en réalité aboutir à un formatage d'un type nouveau. Ainsi, les députés sélectionnés pour leur capacité à refléter la société civile et leur indépendance des contraintes partisanes seraient en contrepartie moins expérimentés et moins familiarisés avec les pratiques de la profession parlementaire, par conséquent plus dociles et plus exposés aux consignes d'un leadership dont le pouvoir a lui-même été renforcé. Le cas de la sélection des candidats parlementaires illustre bien la tension, précédemment évoquée, entre objectifs explicites et objectifs cachés : les réformes conduites au nom d'une "démocratisation" relèvent plutôt d'un travail de "rebranding ", où la décentralisation s'accompagne d'un contrôle accru. Les modifications des règles de sélection des candidats induisent donc des changements organisationnels profonds.

Cependant ces changements de règles du jeu comportent des risques et peuvent produire des effets pervers. Les nouveaux processus (OMOV, primaires ouvertes) échappent parfois au contrôle de la direction nationale et peuvent donner des résultats inattendus, comme l'élection de députés rebelles. L'individualisation du vote et l'adoption du vote postal accentuent en outre l'effet d'atomisation, rendant plus difficile toute coordination d'une action collective, tant par les militants que par le leadership. De plus, il subsiste des risques liés au retrait du «différentiel d'influence » des militants ${ }^{51}$ alors que les partis se reposent largement sur eux pour la conduite de la campagne de terrain, dont l'importance est désormais pleinement reconnue, et en tant que source de revenus.

40 La comparaison entre les deux principaux partis britanniques permet donc, tout en révélant des cultures organisationnelles très différentes, de mettre en lumière les tendances lourdes et les effets de mimétisme. En effet, bien que les mêmes problèmes ne se posent pas dans les mêmes termes et les changements ne se produisent pas au même 
rythme, l'analyse de la sélection des candidats et, plus largement, des réformes organisationnelles à l'œuvre au sein des principaux partis britanniques permet de dessiner une trajectoire commune et de futures pistes de recherche sur la vie politique britannique en particulier et les partis politiques en général.

\section{BIBLIOGRAPHIE}

ALEXANDRE-COLLIER, A. \& AVRIL, E. Les partis politiques en Grande-Bretagne. Paris: Armand Colin, 2013.

GALLAGHER, M. \& MARSH M. Candidate Selection in Comparative Perspective: The Secret Garden of Politics. Londres: SAGE, 1988.

GAUJA, A. "The Individualisation of Party Politics: The Impact of Changing Internal DecisionMaking Processes on Policy Development and Citizen Engagement", The British Journal of Politics and International Relations, vol.17, $\mathrm{n}^{\circ} 1,2015$, pp. 89-105.

HAZAN, R. Y. \& RAHAT, G. Democracies within Parties: Candidate Selection Methods and their Political Consequences. Oxford: Oxford University Press, 2010.

HOPKIN, J. "Bring the Members Back in? Candidate Selection in Britain and Spain", Party Politics, vol. 7, $\mathrm{n}^{\circ}$ 3, 2001, pp. 343-361.

LOW, M. "Parliamentary Candidate Selection in The Conservative Party: The Meaning of Reform For Party Members and Membership Parties”, British Politics, vol. 9, n 4, 2014, pp. 401-429.

MINKIN, L. The Blair Supremacy. A Study in the Politics of Labour's Party Management, Manchester: Manchester University Press, 2014.

PENNINGS, P. \& HAZAN, R. Y. "Democratizing candidate selection. Causes and Consequences", Party Politics, vol. 7, n 3, 2001, pp. 267-275.

SCARROW, S. E. Beyond Party Members. Changing Approaches to Partisan Mobilization. Oxford: Oxford University Press, 2015.

WILLIAMS, R. \& PAUN, A. Party People. How do - and how should - British political parties select their parliamentary candidates. London: Institute for Government,2011 <http:// www.instituteforgovernment.org.uk/sites/default/files/publications/Party\%20People.pdf> [consulté le 18 juin 2015].

\section{NOTES}

1. Pour une évaluation de l'impact des réformes récentes adoptées par les trois partis majoritaires en matière de sélection des candidats, voir par exemple Rhys WILLIAMS \& Akash PAUN, Party People: How do - and how should - British political parties select their parliamentary candidates?, Londres: Institute for Government, 2011 et Peter RIDDELL, Candidate Selection. The Report of the Commission on Candidate Selection, Londres: Electoral Reform Society, 2003.

2. Giovanni SARTORI, Parties and Party Systems, New York: Harper \& Row, 1976. 
3. Reuven Y. HAZAN \& Gideon RAHAT, Democracies within Parties: Candidate Selection Methods and their Political Consequences, Oxford: Oxford University Press, 2010, pp. 6-12.

4. Russell J. DALTON \& Martin P. WATTENBERG, Parties without Partisans. Political Change in Advanced Industrial Democracies, Oxford: Oxford University Press, 2002.

5. Pour une analyse précise des fluctuations de l'adhésion voir Susan E. SCARROW, Changing Approaches to Partisan Mobilization, Oxford: Oxford University Press, 2015, pp. 69-97.

6. Voir par exemple Rémi LEFEBVRE \& Antoine ROGER (dir.), Les partis politiques à l'épreuve des procédures délibératives, Rennes, Presses Universitaires de Rennes, 2009 et James JOHNSON, «Political Parties and Deliberative Democracy?», in Richard S. KATZ \& William CROTTY (eds.), Handbook of Party Politics, Londres: Sage, 2006, pp. 47-51.

7. Reuven Y. HAZAN \& Gideon RAHAT, op. cit.

8. William HAGUE, A Fresh Future for the Conservative Party, Londres: Conservative Central Office, 1997.

9. Peter MAIR, "Party Organisations: from civil society to the state", in Richard S. KATZ \& Peter MAIR (eds.) How Parties Organize: Change and Adaptation in Party Organizations in Western Democracies, Londres: Sage, 1994, pp. 1-22

10. Kenneth CARTY, "Parties as Franchise Systems: The Stratarchical organisational Imperative", Party Politics, vol. 10, $\mathrm{n}^{\circ}$ 1, 2004, pp. 5-24.

11. Mark LOW, "Parliamentary candidate selection in the Conservative Party: the meaning of reform for party members and membership parties", British Politics, vol. 9, n 4, 2014, pp. 401-429. 12. Les assemblées primaires, qui représentent la très grande majorité des primaires ouvertes organisées par le parti conservateur, affichent en moyenne un taux dérisoire de $0,4 \%$ des électeurs inscrits dans la circonscription (entre 100 et 600 participants). Seules les trois primaires postales organisées en 2009 et 2014 à Totnes, Gosport et Rochester and Strood peuvent afficher, notamment pour les deux premières organisées en 2009 , un taux de participation significatif de respectivement $24,5 \%, 17,8 \%$ et $7,6 \%$. Mais le coût d'une primaire postale évalué à $40000 £$ n'a pas permis au parti de poursuivre cette initiative pourtant présentée dans l'accord de coalition de 2010 comme une innovation que le Gouvernement de coalition envisageait d'étendre massivement.

13. L'hypothèse selon Pennings et Hazan est celle d'une plus grande indépendance du député sélectionné par primaire ouverte car il doit sa sélection à l'électorat et non au parti. Or Sarah Wollaston et Caroline Dinenage, les deux députées élues en 2010 après avoir été sélectionnées par primaire postale en 2009, affichaient, dans la précédente législature, un taux de rébellion extrêmement faible par rapport à la moyenne des députés conservateurs (cf. www.publicwhip.org), autrement dit comptaient parmi ceux qui suivaient le plus souvent les consignes de vote du parti.

14. Sources: Paul HUNTER \& Dan HOLDEN, Who Governs Britain? A Profile of MPs in the 2015 Parliament, The Smith Institute, 2015. Voir également les nombreux articles mis en ligne sur le site du projet UK Parliamentary Candidates UK. URL : http://parliamentarycandidates.org

15. David CAMERON, "MPs' expenses: Why I want to open up Tory candidate selection". The Telegraph, 25 May 2009.

16. https://www.conservatives.com/volunteer

17. "If the sitting MP wishes to stand for re-election a trigger ballot will be carried out [...]. If the MP wins the trigger ballot he/she will, subject to NEC endorsement, be selected as the CLP's prospective parliamentary candidate", Labour Party Rule Book 2014, p. 22.

18. Le système de collège électoral, adopté en 1987 sous l'impulsion de Neil Kinnock, avait constitué une première étape dans cette « démocratisation » progressive.

19. Ce point est développé dans Lewis MINKIN, The Blair Supremacy. A Study in the Politics of Labour's Party Management, Manchester University Press, 2014, p. 369. 
20. La " long-list » comprend l'ensemble des candidats éligibles; la " short-list » comprend les finalistes, le plus souvent entre trois et six personnes.

21. "The normal procedure may be dispensed with by the NEC where no valid nominations are received, or when an emergency arises, or when the NEC are of the opinion that the interests of the party would be best served by the suspension of the procedures issued by the NEC". LABOUR PARTY, Labour Party Rule Book2014, p. 23.

22. "A Selection Committee (SC) shall be appointed by the Executive Committee of the CLP in consultation with the NEC Representative". LABOUR PARTY, NEC Procedural Guidance2013, p. 11.

23. "The Selection Committee shall be gender balanced and comprised of not less than six and not more than 10 members. As far as possible it should reflect the demography of the constituency and in particular at least two members representing affiliated organisations". Ibid.

24. Avant 1960, il existait deux listes distinctes, une "A List » (candidats bénéficiant du soutien d'un syndicat) et une "B List» (autres). En 1960, ces deux listes ont été fondues en une liste nationale unique (voir Eric SHAW, Discipline and Discord in the Labour Party, 1987). A l'heure actuelle, le NEC compile une liste appelée « National Parliamentary Panel » qui inclut des candidats approuvés par les organisations affiliées. Les candidats sélectionnés en dehors de cette liste doivent se soumettre à un entretien avant de recevoir l'approbation du NEC.

25. "The NEC shall have a supervisory role". op. cit., p. 4.

26. Dans les sièges ordinaires, un minimum de quatre candidats dont au moins deux femmes ; dans les sièges appliquant les « AWS ", un minimum de trois candidats.

27. "[...] to ensure that objectives in relation to quality and diversity are met. This procedure is designed to ensure the process is open and accessible to candidates from all backgrounds including individuals from under-represented groups". op. cit., p. 4.

28. "The selection of a parliamentary candidate is subject to the endorsement by the NEC". Ibid., p. 4.

29. "Disputes arising out of the selection procedure shall be considered by an officer appointed by the NEC who shall report to them. The NEC's decision on that report shall be final and binding on all parties for all purposes". Labour Party Rule Book 2014, p. 25.

30. "[...] to select more candidates who reflect the full diversity of our society in terms of gender, race, sexual orientation and disability, and to increase working class representation". NEC Procedural Guidance 2013, p. 4.

31. Sur cette dimension voir par exemple Pippa NORRIS \& Jon LOVENDUSKI, Political Recruitment. Gender, Race and Class in the British Parliament, Cambridge: Cambridge University Press, 1994.

32. Sur la «docilité » des députées travaillistes, voir Philip COWLEY \& Sarah CHILDS, «Too Spineless to Rebel? New Labour's Women MPs", British Journal of Political Science, vol. 33, n 3,pp. 345-365.

33. Sur l'évolution du sélectorat, voir par exemple Paul WEBB, The Modern Party System, Londres : Sage, 2000, pp. 207-208.

34. "All members participate in the final selection of the candidate in an OMOV ballot, either at hustings meetings or by post must be eligible members of the Labour Party", LABOUR PARTY, NEC Procedural Guidance2013, p. 11.

35. Michael GALLAGHER \& Michael MARSH, Candidate Selection in Comparative Perspective, Londres: Sage, 1988, p. 3.

36. "[...] be a current member of the constituency in question at the freeze date and at each stage of the procedure", Procedural Guidance 2013, p. 12.

37. "Have at least six months continuous membership of the Labour Party (any constituency) at the freeze date", Ibid.

38. Anna B. MIKULSKA et Susan E. SCARROW soulignent très justement l'importance à accorder aux détails des procédures qui se cachent derrière les discours officiels de "démocratisation ", dans "Assessing the Political Impact of Candidate SelectionRules: Britain in the 1990s", Journal of Elections, Public Opinion and Parties, vol. 20, n 3, pp. 311-333, août 2010. 
39. Voir par exemple Patrick WINTOUR, "MP condemns Labour decision to increase influence over selection of parliamentary candidates", The Guardian, 20 novembre 2014. URL: http://www.theguardian.com/politics/2014/nov/20/tom-watson-condemns-labourleader-influence-parliamentary-candidate-selection[consulté le 15/05/15].

40. Peter MAIR, dans un article de 1994 abondamment cité depuis (cf. supra), avait souligné que l'extension de droits électifs à l'ensemble des membres visait à marginaliser les militants, moins dociles.

41. Voir Patrick SEYD \& Paul WHITELEY, High-intensity Participation: The Dynamics of Party Activism in Britain, Ann Arbor :The University of Michigan Press, 2002, p. 23.

42. Lewis MINKIN, op. cit., p. 370.

43. Ibid. et Eric SHAW, op. cit. Ces formes de pression, qui ne peuvent être relevées que par le biais d'une observation directe, ont été développées dans Emmanuelle AVRIL, Du Labour au New Labour. Le changement vue de l'intérieur, Villeneuve d'Ascq :Presses du Septentrion, 2007.

44. Sources: Paul HUNTER \& Dan HOLDEN, Who Governs Britain? A Profile of MPs in the 2015 Parliament, The Smith Institute, 2015.Voir également les nombreux articles mis en ligne sur le site du projet UK Parliamentary Candidates UK. URL : http://parliamentarycandidates.org

45. Il n'y a pas d'élection dans la circonscription représentée par le président de séance de la Chambre des Communes ( Speaker »), qui est travailliste.

46. Le parti travailliste a remporté un total de 232 sièges, contre 258 en 2010.

47. Sur les "AWS" voir Rosie CAMPBELL, "All women shortlists remain a controversial but effective way to improve women's representation in politics", The Constitution Unit, 5 février 2015. URL: http:// parliamentarycandidates.org/news/all-women-shortlists-remain-a-controversial-but-effectiveway-to-improve-womens-representation-in-politics/

48. Sur ce point, voir la critique de Peter WATT, ancient secrétaire général du parti travailliste (2006-2007), dans « Labour's new way of selecting parliamentary candidates makes a mockery of calls for more working class MPs", Labour Uncut, 8 février 2013. URL: http://labouruncut.co.uk/2013/02/08/labours-new-way-of-selecting-parliamentary-candidates-makes-amockery-of-calls-for-more-working-class-mps/ et celle publiée par le député Michael MEACHER sur son blog: "The alienateduntouchables", 19 avril 2015. URL: http:// www.michaelmeacher.info/weblog/2015/04/the-alienated-untouchables/

49. Voir Agnès ALEXANDRE-COLLIER \& Emmanuelle AVRIL, Les partis politiques en Grande-Bretagne, Paris: Armand Colin, 2013.

50. Richard KATZ \& Peter MAIR, "Changing Models of Party Organization and Party Democracy: the Emergence of the Cartel Party", Party Politics, vol. 1, n 1, 1995, pp. 5-28; Richard KATZ \& Peter MAIR, "Party Organisation, Party Democracy and the Emergence of the Cartel Party", in Peter MAIR (ed.),Party System Change: Approaches and Interpretations, Oxford: Oxford University Press, 1997, pp. 93-119.

51. Jonathan HOPKIN, "Bringing the Members Back In?: Democratizing Candidate Selection in Britain and Spain", Party Politics, vol. 7, n 3, mai 2001, pp. 343-361.

\section{RÉSUMÉS}

La sélection des candidats parlementaires aux élections législatives est l'une des fonctions les plus cruciales qui incombent aux sections locales, lesquelles ont longtemps joui, dans ce 
domaine, d'une relative autonomie par rapport au siège des partis. Mais partant du principe que l'agrégation des choix individuels des sections locales détermine la composition du groupe parlementaire, la direction nationale de chacun des partis a conçu de nouvelles stratégies et règles leur permettant d'influencer ces choix, l'objectif étant de s'assurer que les futurs députés seront plus conformes à la ligne définie par la direction du parti et la composition sociodémographique du groupe parlementaire plus représentative de l'électorat. Cet article étudie donc l'impact de ces procédures de sélection à la fois sur l'organisation partisane et la représentativité des groupes parlementaires travaillistes et conservateurs issus des élections.

The selection of parliamentary candidates for the general election is one of the most crucial tasks carried out by local parties who have long enjoyed relative autonomy from party headquarters in this respect. But because the aggregate choices of individual local parties determine the composition of the parliamentary parties, the party leaderships in both parties have devised new strategies and rules allowing them to influence that choice. This is in order both to ensure both that future MPs are more in tune with the leadership line and that the socio-demographic composition of the parliamentary parties is more representative of the electorate. This article therefore looks at the impact of the methods of candidate selection both on party organization and on the representativeness of the Conservative and Labour parliamentary parties which has emerged out of the election.

INDEX

Keywords : Conservative party, Labour party, candidate selection, local parties, primaries

Mots-clés : parti conservateur, parti travailliste, sélection des candidats, section locales, primaires

\section{AUTEURS}

\section{AGNÈS ALEXANDRE-COLLIER}

Université de Bourgogne

EMMANUELLE AVRIL

Université Sorbonne Nouvelle - Paris 3 